\title{
ESBL-Producing E. Coli in a Patient on Automated Peritoneal Dialysis
}

\section{Faqi $\mathrm{MK}^{* 1}$, Ebrahim $\mathrm{MA}^{2}$ and $\mathrm{Al}$ Ghareeb SM${ }^{3}$}

${ }^{1}$ Orthopedic Resident, Department of Orthopedics, Royal Medical Services, Bahrain Defence Force Hospital, Riffa, Bahrain

${ }^{2}$ Medical Intern, Salmaniya Medical Complex, Bahrain

${ }^{3}$ Consultant Nephrologist and Chairperson of the Nephrology and Transplant Department at the Salmaniya Medical Complex, Faculty of Medicine and Medical Sciences, Arab Gulf University, Kingdom of Bahrain

*Corresponding author: Faqi MK, Orthopedic Resident, Department of Orthopedics, Royal Medical Services, Bahrain Defence Force Hospital, Riffa, Bahrain, Tel: 0097366666717, E-mail: drmfaqi@gmail.com

Citation: Faqi MK, Ebrahim MA, Al Ghareeb SM (2016) ESBL-Producing E. Coli in a Patient on Automated Peritoneal Dialysis. J Case Rep Stud 4(1): 106. doi: 10.15744/2348-9820.4.106

Received Date: December 02, 2015 Accepted Date: February 12, 2016 Published Date: February 16, 2016

\begin{abstract}
Peritoneal Dialysis (PD) is one of the 3 well-established modalities of renal replacement therapy used in patients with renal failure. Despite its significant role as a successful method of renal replacement therapy, $\mathrm{PD}$ is highly associated with peritonitis and catheterrelated infections. ESBL-producing E. coli (ESBL-EC) is uncommonly isolated in the peritoneal dialysate and are resistant to the currently recommended empiric antibiotics for peritoneal dialysis peritonitis (PDP). The optimal treatment of ESBL-EC cultured in the peritoneal dialysate is still lacking. Here, we report a case of 68 years old female on automated peritoneal dialysis, who presented with symptoms of peritonitis, with a peritoneal dialysate culture positive for ESBL-EC.

Keywords: Automated peritoneal dialysis; End stage renal disease; Escherichia coli; Extended spectrum beta lactamase (ESBL); peritonitis

List of Abbreviations: APD: Automated Peritoneal Dialysis; CAPD: Continuous ambulatory peritoneal dialysis; CLSI: Clinical and laboratory standards institute; CRP: C-reactive protein; EC: E. coli; ESBL: Extended Spectrum Beta-Lactamase; ESR: Erythrocyte Sedimentation Rate; ESRD: End stage renal disease; HCV: Hepatitis C Virus; IP: Intraperitoneal; ISPD: International Society of peritoneal dialysis; PD: Peritoneal Dialysis; PDF: Peritoneal dialysate fluid; PDP: Peritoneal dialysis peritonitis; PMN: Polymorphonucleocytes; RCT: Randomized Controlled Trial; UAE: United Arab Emirates; WBC: White Blood Cells
\end{abstract}

\section{Introduction}

End-stage renal disease (ESRD) is a major cause of morbidity and mortality. Worldwide, an estimated 10-15\% of patients with ESRD are on peritoneal dialysis (PD) [1]. Despite its significant role as a successful method of renal replacement therapy, PD is highly associated with peritonitis and catheter-related infections. Patients on PD, regardless of febrile status, who complain of abdominal pain associated with a cloudy dialysate fluid, are highly suspected of peritonitis. A White Blood Cell (WBC) count greater than $100 \mathrm{~mm}^{3}$ comprising more than 50\% polymorphonucleocytes (PMNs) confirms the diagnosis and entails immediate treatment [2].

The International Society for Peritoneal Dialysis (ISPD) recommends an empiric antibiotic therapy covering both gram positive and gram negative organisms for the treatment of peritonitis in patients on PD. Antibiotic selection is to be made according to the Center's common causative organisms, keeping in mind sensitivity issues. Causative organisms and sensitivity issues differ from country to another [3].

ISPD also recommends a two/three weeks' treatment of intraperitoneal (IP) cephalosporin (e.g. ceftazidime or cefepime) for E. coli related peritonitis [3].

ESBL-producing E. coli (ESBL-EC), an uncommonly isolated in the peritoneal dialysate, is resistant to cephalosporins, penicillins and aztreonam, as per the clinical and laboratory standards institute (CLSI) recommendations [4]. A standard protocol for ESBLEC treatment does not currently exist.

Since ESBL producing E. coli peritonitis produced higher mortality rates than non-ESBL producing E. coli (27.3\% vs $3.9 \% \mathrm{p}=0.02)$ [2], presence of ESBL-EC in the peritoneal dialysate introduces a new challenge in choosing effective antibiotics. The antibiotics most effective against ESBL-Ec in the peritoneal dialysate has not been established and requires further investigations. 


\section{Case Presentation}

A 68-year-old Bahraini Female known to have ESRD and one-year on Automated Peritoneal Dialysis (APD), presented to the Accident and Emergency Department complaining of a one-day history of generalized abdominal pain and constipation. She had a long history of uncontrolled Diabetes Mellitus Type 2, hypertension and Hepatitis C Virus (HCV). On admission, she was afebrile with a temperature of $36.6^{\circ} \mathrm{C}$, heart rate of 105 beats/min and blood pressure of $94 / 59 \mathrm{mmHg}$. Physical examination findings were notable for diffuse abdominal tenderness and distention. No signs of infection were seen at the peritoneal dialysis catheter exit site. Examination was otherwise unremarkable. A complete blood count (CBC) was notable for a WBC count of $14.8 \times 10^{\wedge} 9 / \mathrm{L}$ and $86 \%$ polymorphs on differential. Presuming bacterial peritonitis, the patient was empirically treated with IP ceftazidime $1.5 \mathrm{~g}$ once daily and IP vancomycin $2 \mathrm{~g}(30 \mathrm{mg} / \mathrm{kg})$ every 5 days.

Chest, abdominal and kidney-ureters-bladder radiographs and an Electrocardiogram were normal. Abdominal Ultrasound revealed a large amount of anechoic free fluid filling the abdominal cavity; consistent with ascites. Blood and mid-stream urine cultures were sterile after 48 hours of incubation. Erythrocyte sedimentation rate (ESR) was $80 \mathrm{~mm} / \mathrm{hr}$ and C-reactive protein (CRP) was $111 \mathrm{mg} / \mathrm{L}$. Peritoneal dialysate was turbid with a WBC count of $11800 / \mu \mathrm{L}, 80 \%$ were neutrophils, and no eosinophils were detected. Using the double disk diffusion method based on the Clinical Laboratory Standards Institute guidelines [4], the difference in diameters of inhibition zones with ceftazidime versus amoxicillin-clavulanate was $\geq 5 \mathrm{~mm}$; therefore, presence of ESBL-producing E. coli in the peritoneal dialysate effluent was confirmed. The cultured ESBL-EC strain's sensitivity profile (antibiogram) is shown in Table 1.

\begin{tabular}{|c|c|}
\hline Antibiotic & Susceptibility \\
\hline Tigecycline & Sensitive \\
\hline Augmentin & Resistant \\
\hline Cefuroxime-oral & Resistant \\
\hline Cefuroxime IV & Resistant \\
\hline Ceftriaxone & Resistant \\
\hline Cefipime & Resistant \\
\hline Meropenem & Sensitive \\
\hline Cotrimoxazole & Resistant \\
\hline Gentamicin & Sensitive \\
\hline Ciprofloxacin & Sensitive \\
\hline
\end{tabular}

Table 1: Antimicrobial susceptibility profile of the dialysate cultured ESBL-E. coli strain

Based on the sensitivity profile shown in Table 1, the IP Ceftazidime and Vancomycin were discontinued and Meropenem was started. On day 3, the patient's symptoms improved and a clear peritoneal dialysate fluid showed a drop in WBC to 480 with $90 \%$ polymorphs. The patient was discharged on the same day and advised to continue the full course of antibiotics. Serial dialysate fluid analyses and cultures were done on follow-up and are shown in Table 2. Dialysate cultures were sterile on follow up. Other laboratory indices were of normal limits.

\begin{tabular}{|c|c|c|}
\hline Day & White blood cell count & Polymorph count (\%) \\
\hline 3 & 480 & 90 \\
\hline 6 & 900 & 90 \\
\hline 7 & 280 & 85 \\
\hline 8 & 68 & 76 \\
\hline 10 & 18 & Nil \\
\hline 13 & Nil & Nil \\
\hline
\end{tabular}

Table 2: Serial white blood cells (WBC) and polymorph counts of the dialysate fluid according to hospitalization day

The patient continued on APD with no complications or acute events until she developed the same symptoms 8 months later, where she was admitted to the medical ward. A CBC showed a WBC count of $13.210^{\wedge} 9 / \mathrm{L}, 86 \%$ of which were polymorphs. She was empirically started on Meropenem based on the susceptibility profile of the previously cultured ESBL-EC. A peritoneal dialysate fluid (PDF) analysis showed turbid fluid with a WBC count 400, 80\% of which were polymorphs. CRP was 71.8 . The microbiological cultures of the PDF obtained prior to starting Meropenem were similar to the previous admission (Table 1). After 5 days, PDF analysis showed a WBC count 10 with no polymorphs. A PDF culture was sterile. Based on these findings, and because her symptoms gradually subsided, the antibiotics were discontinued and the patient was discharged. Peritoneal equilibrium tests (PET) were not done for the patient. 


\section{Discussion}

Peritoneal Dialysis (PD) is one of the 3 well-established modalities of renal replacement therapy used in patients with renal failure. It is used in more than $11 \%$ of the global dialysis population [5]. The number of patients on PD increased by 21.8 and 24.9 patients per million population in both developed and developing countries, respectively [6]. It has superior therapeutic outcomes to hemodialysis in the first few years of treatment [5]. Also, it has been associated with better preservation of residual renal function; less ischemic kidney insult and avoidance of the damaging inflammatory effects of extracorporeal dialysis [6]. Nevertheless, peritonitis remains a common complication of PD leading to high incidence of morbidity and mortality, with a global incidence ranging from 0.06 to 1.66 episodes per patient-year. It is estimated that $60 \%$ of patients on continuous ambulatory peritoneal dialysis (CAPD) will develop peritonitis in the first year and $80 \%$ in the first 30 months [5,7]. Peritoneal Dialysis Peritonitis (PDP) can lead to technique failure (20\%), ultrafiltration failure, catheter removal, encapsulating peritoneal sclerosis and death (2-6\%) $[5]$.

The causative organisms of PDP are mainly gram-positive cocci (coagulase-negative Staphylococcus epidermidis and Staphylococcus aureus), and gram-negative organisms such as E-coli. E. coli is the most common gram-negative causative pathogen, with a report prevalence of up to $59 \%$ of gram-negative PDP in some centers $[2,6,8,9]$. Despite the reduction in incidence of PDP through the use of prophylactic antibiotics, risk factors management and advances in PD techniques, the incidence of gram-negative PDP has not changed [6]. Moreover, an increased recognition of ESBL producing Enterobacteriaceae causing PDP has been reported [6,8].

ESBL incidence has been rising globally with reported rates of 3.3-4.7\% in North America and 6.7-25.4\% in South America [2]. Much higher rates were reported in the Arabian Gulf. In Kuwait 31.7\% of Enterobacteriaceae were ESBL producing and 41\% of inpatient isolates in the United Arab Emirates (UAE), with E-coli being the most common ESBL-producing pathogen in both countries [10]. The incidence of ESBL-producing organisms in PDP in Bahrain has not been reported, we report the first case of ESBL-producing E.coli (ESBL-EC) PDP in the country.

ESBL isolates in PDP were not previously a common finding. However, its incidence highly depends on the prevalence of ESBL in the dialysis providing institution. In 2014, Parsada et al. reported one of the highest rates of ESBL PDP in India, with 54.3\% of PDP Enterobacteriaceae isolates being ESBL-producers [1]. On the other hand, out of 125 gram-negative PDP patients, a retrospective study in Southern Taiwan reported only 1 case of ESBLs PDP between 1990 to 2011 [9].

The true importance of ESBLs (including ESBL Peritonitis and PDP) lies within the well-established increased mortality and morbidity associated with these infections. This is mainly attributed to the late recognition and delay of appropriate antibiotics treatment $[1,2,7,8,10,11]$. A case control study of 31 ESBL-EC and $K$. pneumonia bacteremia found that less mortality occurred in those receiving appropriate treatment within 3 days from bacteremia [2]. A retrospective case control study investigated patients suffering from spontaneous bacterial peritonitis (SBP) between Jan 2000 and Dec 2006. 368 episodes in 346 patients were caused by E.coli and K.pneumonia. Poorer outcomes (including death) were significantly higher in those with ESBL producing isolates in comparison to those without it [11]. Parsade et al. examined 303 PDP in 211 patients and found that ESBLs accounted for 54.3\% of Enterobacteriaceae and a significantly higher mortality rate was observed in such patients [1]. Yip et al reported 11 cases of ESBL producing pathogens in a sample of 88 episodes of $E$. coli in CAPD peritonitis patients over a 10-year period. Peritonitis secondary to ESBLs was associated with higher rates of treatment failure $(45.5 \%$ vs. $13.0 \%, \mathrm{p}=0.02)$ and death secondary to sepsis $(27.3 \%$ vs. $3.9 \%, \mathrm{p}=0.02)[2]$.

In spite of the high mortality associated with ESBL infections, the relatively low incidence of such organisms in PDP patients have discouraged the empiric use of antibiotics that are effective against ESBLs in the mainstay management of PDP. Research towards establishing the risk factors for the presence of ESBL organisms in PDP have been encouraged to reduce the incidence of the disease and to help identify and selectively treat high risk patients [11]. Yip et al. suggested that the previous use of cephalosporin antibiotics and gastric acid inhibitors are major risk factors for developing ESBLs in CAPD Peritonitis patients [2]. The use of H2 antagonists is associated with increased intragastric bacteria [2,12-15]. Gastric acids prevent bacterial colonization in the small bowel and thus prevent intestinal infections. Also, Caravaca et al. found that peritonitis secondary to enteral origin is associated with the inhibition of gastric acid [2,16]. A retrospective study of 247 cases of PDP found that resistant bacterial peritonitis was significantly associated with the use of antimicrobial therapy in the last 3 months [17]. Other risk factors include longer hospitalization and the admission to chronic care facilities [7]. Furthermore, Feng et al. (2014) reported 68 cases of E. coli PDP, $35.5 \%$ of which were ESBL producing. They concluded that patients with a previous history of peritonitis were at a significantly higher risk of ESBL PDP compared to those with no prior history [odds ratio (OR): 5.286; 95\% confidence interval (CI): 2.018 13.843; $\mathrm{p}=0.001$ ] [8]. In our case report, the patient had a previous history of peritonitis, multiple episodes of hospital admissions and a recent history of omeprazole use, all of which were risk factors for developing ESBL peritonitis. Hence, the decision was made to start empiric Meropenem during her second admission. 
The high number of patients on PD, the worldwide increase in incidence of gram-negative PDP and the rise in ESBL-producing enterobacteriaceae infection along with the higher mortality and morbidity associated with such infections are factors that emphasize the need for establishing protocols and guidelines for the treatment of ESBL-producing peritonitis in PD patients. Current international guidelines and treatment protocols only recommend for the optimal treatment of non-ESBL-producing PDP, whilst evidence is lacking for the treatment of ESBL-producing PDP. The International Society of Peritoneal Dialysis (ISPD) recommends the empiric treatment of PDP with antibiotics that "cover both gram-positive and gram-negative organisms guided by local antimicrobial sensitivity and resistance". Vancomycin or first generation cephalosporins for gram-positive organisms, while a third-generation cephalosporin or an aminoglycoside for gram-negative coverage [3]. It is also recommended that the antibiotic selection be made according to the centers own pattern of infectious organisms and susceptibility profile, whilst keeping in mind the patient's previous history of infectious organisms [3]. Such recommendations highlight the importance of monitoring infection rates and microbiological profile to determine the PD providing institution's best choice of empiric treatment $[3,5,18,19]$. Based on current literature, the ISPD concludes that no single empirical combination of antibiotics is superior to another $[3,18]$. However, vancomycin is superior in cases of presence (or a history of) methicillin-resistant Staphylococcus aureus (MRSA) [3,20]. On the other hand, a recent proportional meta-analysis of case series done in 2014 identified a superiority of a glycopeptide (vancomycin) and ceftazidime empiric regimen [19]. This was explained by the global increase in pseudomonas resistance and thus increasing effectiveness of ceftazidime. However, precaution is to be made when using such recommendation because the meta-analysis included only one randomized control trial (RCT) [19]. Finally, a combination of oral ciprofloxacin and IP cefazolin was recommended as a cost-effective empirical first choice [21].

Bindayna et al. (2009) was the first to investigate the rate of ESBL in Bahrain. A total of 11,886 Enterobacteriaceae were isolated over a 2 -year period, $22.6 \%$ of which were ESBL producers, $52.2 \%$ were E. coli and $87.7 \%$ were from inpatients. However, most of the ESBL-producing pathogens were found in urinary infections [10]. In our case, the use of the combination of vancomycin and ceftazidime was based on the hospital's high rates of MRSA and Pseudomonas infections. However, because ESBLs are highly resistant to ceftazidime, such regimen lacks effectiveness against ESBL PDP. Therefore, and due to the high prevalence of ESBL in Bahrain, identifying high-risk cases of ESBL PDP is essential to decrease mortality. This also entails the need for investigating the most effective empiric antimicrobial therapy for patients who are high risk for developing ESBL PDP.

Although a consensus on the appropriate empirical antimicrobial therapy for ESBL PDP has not been made, most published case reports identified their ESBLs as carbapenam sensitive. Similarly, Bindayna et al. (2009) reported ESBLs in Bahrain as susceptible to both imipenem and meropenem [10]. Carbapenam resistance was rarely reported in literature. The first case was identified in 1999, whilst only one case was ever reported in the Arabian Gulf region in UAE (2007) [10].

Other antibiotics that are highly potent against ESBL include tigecycline and polymyxin $\mathrm{B}$. Although in-vitro studies of tigecycline showed a lack of cross-resistance to ESBLs [22], a pooled analysis of 13 clinical trials showed that it is associated with higher mortality rates compared with patients receiving comparator antibacterial used in Intra-abdominal infections including peritonitis, rendering it ineffective in severe infections [23]. On the other hand, polymyxin B has a unique high activity against all multidrug-resistant gram-negative organisms except Proteus species and Serratia marcescens. A case report of K. pneumoniae PDP showed rapid response to polymyxin B after a 1 week persistent peritonitis despite catheter removal and a combination regimen of meropenem and amikacin [7]. However, polymyxins have a large molecular size and therefore don't easily penetrate pleural membranes. Furthermore, since the kidneys of patients on PD are readily damaged, the association of polymyxins with nephrotoxicity is of little importance [7]. Finally, amikacin is a possible alternative, but its use is limited because of its side effect profile, especially in children $[7,20]$.

The ISPD recommends an extended period of treatment for severe and gram negative PDP, typically a total of 3 weeks [3,5]. The intraperitoneal route has proven to be superior to intravenous but similar to oral, although all RCTs available looked at ciprofloxacin as the oral agent [5]. Our patient was given IP Meropenam for a total of 2 weeks only which proved as seen table 2 to be very effective. On the other hand, switching patients on APD to CAPD during the time of treatment or increasing the time of the cycler remains a controversy. Although lacking evidence, Cho et al. hypothesize that the short dwells in APD may lead to slower antibiotic absorption and faster clearance. Based on such hypotheses, they suggest either resetting the cycler to longer dwells or switching patients to CAPD [5]. However, research is still needed to ascertain which has a better outcome. Finally, ISPD also suggest the removal of the PD catheter in resistant peritonitis (i.e. if the effluent fails to clear after 5 days of appropriate antibiotic treatment), relapsing peritonitis, and fungal peritonitis. No recommendations for ESBLs exist [3,5].

\section{Conclusion}

ESBL-producing organisms are increasingly seen in the peritoneal fluids of PDP patients. The current recommended empirical therapy for the management of PDP lacks effectiveness against such organisms. With such increased incidence, future research should focus on identifying criteria for the use of an empiric antibiotic regimen that is effective against ESBL-producing organisms in high-risk patients. Also, the optimal treatment of ESBL-EC in PDP should be investigated. 


\section{References}

1. Prasad KN, Singh K, Rizwan A, Mishra P, Tiwari D, et al. (2014) Microbiology and outcomes of peritonitis in northern India. Perit Dial Int 34: 188-94.

2. Yip T, Tse KC, Lam MF, Tang S, Li FK, et al. (2006) Risk factors and outcomes of extended-spectrum beta-lactamase-producing E. coli peritonitis in CAPD patients. Perit Dial Int 26: 191-7.

3. Li PK, Szeto CC, Piraino B, Bernardini J, Figueiredo AE, et al. (2010) Peritoneal dialysis-related infections recommendations: 2010 update. Perit Dial Int 30: 393-423.

4. Clinical and Laboratory Standards Institute (2013) Performance standards for antimicrobial susceptibility testing; Twenty-third informational supplement. CLSI document M100-S23. Clinical Laboratory Standards Institute 33: 1-188.

5. Cho Y, Johnson DW (2014) Peritoneal dialysis-related peritonitis: towards improving evidence, practices, and outcomes. Am J Kidney Dis 64: 278-89.

6. Li PK, Chow KM (2013) Peritoneal dialysis-first policy made successful: perspectives and actions. Am J Kidney Dis 62: $993-1005$.

7. Parchuri S, Mohan S, Cunha BA (2005) Extended spectrum beta-lactamase-producing Klebsiella pneumoniae chronic ambulatory peritoneal dialysis peritonitis treated successfully with polymyxin B. Heart Lung 34: 360-3.

8. Feng X, Yang X, Yi C, Guo Q, Mao H, et al. (2014) Escherichia coli Peritonitis in peritoneal dialysis: the prevalence, antibiotic resistance and clinical outcomes in a South China dialysis center. Perit Dial Int 34: 308-16.

9. Lin WH, Tseng CC, Wu AB, Yang DC, Cheng SW, et al. (2015) Clinical and microbiological characteristics of peritoneal dialysis-related peritonitis caused by Klebsiella pneumoniae in southern Taiwan. J Microbiol Immunol Infect 48: 276-83.

10. Bindayna KM, Senok AC, Jamsheer AE (2009) Prevalence of extended-spectrum beta-lactamase-producing Enterobacteriaceae in Bahrain. J Infect Public Health 2: 129-35.

11. Song KH, Jeon JH, Park WB, Park SW, Kim HB, et al. (2009) Clinical outcomes of spontaneous bacterial peritonitis due to extended-spectrum beta-lactamaseproducing Escherichia coli and Klebsiella species: a retrospective matched case-control study. BMC Infect Dis 9: 41.

12. Stockbrugger RW, Cotton PB, Eugenides N, Bartholomew BA, Hill MJ, et al. (1982) Intragastric nitrites, nitrosamines, and bacterial overgrowth during cimetidine treatment. Gut 23: 1048-54.

13. Reusser P, Zimmerli W, Scheidegger D, Marbet GA, Buser M, et al. (1989) Role of gastric colonization in nosocomial infections and endotoxemia: a prospective study in neurosurgical patients on mechanical ventilation. J Infect Dis 160: 414-21.

14. Cristiano P, Paradisi F (1982) Can cimetidine facilitate infections by oral route. Lancet 2: 45.

15. Wingate DL (1990) Acid reduction and recurrent enteritis. Lancet 335: 222.

16. Caravaca F, Ruiz-Calero R, Dominguez C (1998) Risk factors for developing peritonitis caused by micro-organisms of enteral origin in peritoneal dialysis patients. Perit Dial Int 18: 41-5.

17. Kofteridis DP, Valachis A, Perakis K, Maraki S, Daphnis E, et al. (2010) Peritoneal dialysis-associated peritonitis: clinical features and predictors of outcome. Int J Infect Dis 14: e489-93.

18. Wiggins KJ, Johnson DW, Craig JC, Strippoli GF (2007) Treatment of peritoneal dialysis-associated peritonitis: a systematic review of randomized controlled trials. Am J Kidney Dis 50: 967-88.

19. Barretti P, Doles JV, Pinotti DG, El Dib R (2014) Efficacy of antibiotic therapy for peritoneal dialysis-associated peritonitis: a proportional meta-analysis. BMC Infect Dis 14: 445 .

20. Caring for Australians with Renal Impairment (2004) The CARI guidelines. Evidence for peritonitis treatment and prophylaxis: treatment of peritoneal dialysisassociated fungal peritonitis. Nephrology (Carlton) 3: S78-81.

21. Lima RC, Barreira A, Cardoso FL, Lima MH, Leite M (2007) Ciprofloxacin and cefazolin as a combination for empirical initial therapy of peritoneal dialysisrelated peritonitis: five-year follow-up. Perit Dial Int 27: 56-60.

22. Morosini MI, García-Castillo M, Coque TM, Valverde A, Novais A, et al. (2006) Antibiotic coresistance in extended-spectrum-beta-lactamase-producing Enterobacteriaceae and in vitro activity of tigecycline. Antimicrob Agents Chemother 50: 2695-9.

23. Blot S, De Waele JJ, Vogelaers D (2012) Essentials for selecting antimicrobial therapy for intra-abdominal infections. Drugs 72: e17-32.

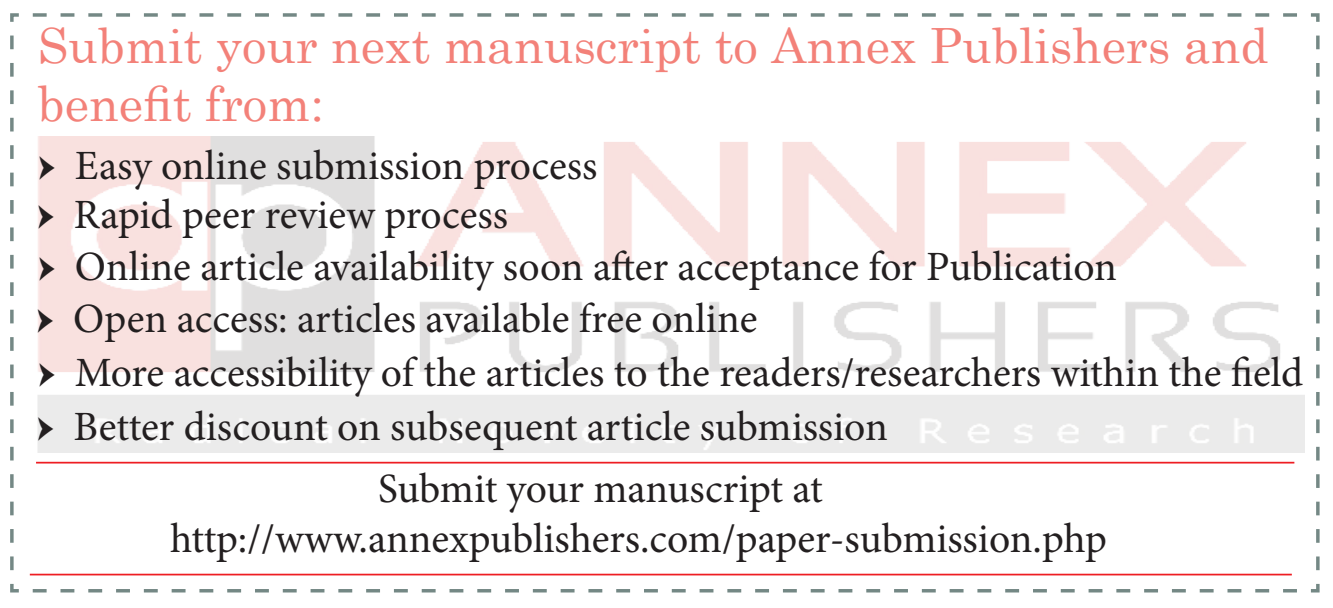

